\title{
A Wave Propagation Model for a Terahertz Single Pixel Camera
}

\author{
J. Hieronymus ${ }^{1}$, S. Augustin ${ }^{1,2}$, and H.-W. Hübers ${ }^{1,2}$ \\ ${ }^{1}$ German Aerospace Center (DLR), Institute of Optical Sensor Systems, Rutherfordstr. 2, 12489 Berlin, Germany \\ ${ }^{2}$ Humboldt-Universität zu Berlin, Department of Physics, Newtonstr. 5, 12489 Berlin, Germany
}

\begin{abstract}
Spatially resolved terahertz measurements usually require either mechanical scanning, large and expensive detector arrays, or a combination thereof. So called single pixel cameras (SPCs), consisting mainly of a spatial light modulator (SLM) and a single pixel detector, provide an alternative approach. The SLM is a dynamically reconfigurable spatial filter. For a successful image acquisition, the setup, and especially the behaviour of the SLM, needs to be physically well-understood. According to the abovementioned requirements, a wave propagation model for a transmission imaging configuration was developed. It is capable of predicting signal values at the single pixel detector for a given wavelength, spatial filter configuration and detector position. The developed model is presented here and verified using a $0.345 \mathrm{THz}$ SPC setup.
\end{abstract}

\section{INTRODUCTION AND EXPERIMENTAL SETUP}

$\mathrm{T}$ HE analysed single pixel camera (SPC) consists of a $0.345 \mathrm{THz}$ multiplier source (Tx), a collimating TPX lens, a $\mathrm{THz}$ spatial light modulator (SLM) and a heterodyne receiver $(\mathrm{Rx})$ as detector. Due to its principles of operation, the $\mathrm{THz}$ source emits coherent radiation. The THz SLM is a disc of germanium, which can be optically tuned to transmit more or less $\mathrm{THz}$ radiation by shining VIS/NIR light onto it. This light is emitted by a $250 \mathrm{~W}$ tungsten halogen lamp and then spatially structured by a Digital Micromirror Device (DMD), which is a SLM for VIS/NIR light. The DMD has $1024 \times 768$ micromirrors, which can be independently controlled. The micromirrors are optically mapped onto the surface of the germanium disc. Fig. 1 depicts the setup.

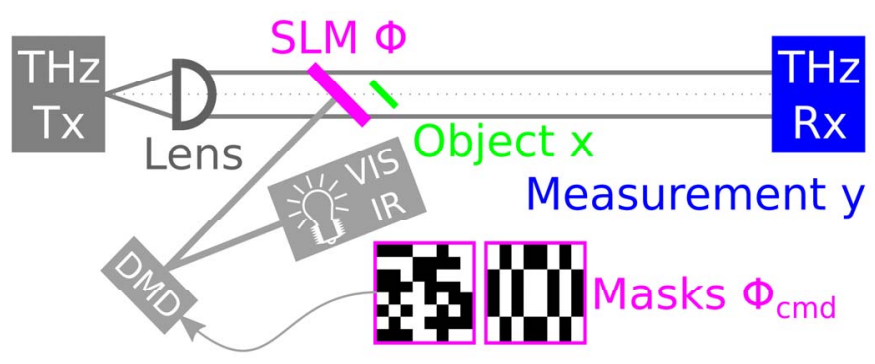

Fig. 1. Schematic drawing of the SPC setup.

DMD: Digital Micromirror Device (SLM for visible light).

The process of image acquisition is realised as follows: An object $(\mathrm{x})$ to be imaged is placed into the collimated beam of the $\mathrm{THz}$ source $(\mathrm{Tx})$. A set of spatial filter configurations (masks $\Phi_{\mathrm{cmd}}$ ) is commanded and the corresponding detector signals (y) are measured. The resulting THz spatial filter $(\Phi)$ is strongly correlated with the commanded mask, but not identical. One of the reasons for this is the depth of modulation that is below $100 \%$. The object $\mathrm{x}$ is then to be reconstructed from the known spatial filter configurations $\Phi_{\mathrm{cmd}}$ and the measured detector signals y. In the simplest case, the reconstruction algorithm assumes $\Phi=\Phi_{\mathrm{cmd}}$ and follows
$\mathrm{x}=\Phi^{-1} \cdot \mathrm{y}$, see $[1,2]$. Imaging an "empty object", i.e. no objects are present in the $\mathrm{THz}$ beam, reveals some properties of a given SPC. One of the most obvious outcomes is the presence of a circular artefact. (cf. Fig. 2). An important research goal was to identify the origin of this artefact.

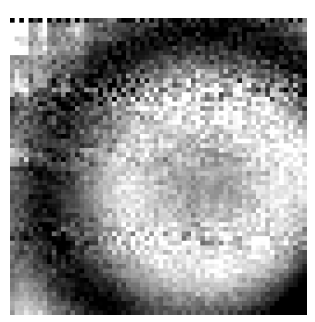

Fig. 2. SPC image reconstruction of the "empty object". The dark circular structure is the artefact to be analysed.

\section{SimUlation MODEL}

When imaging the "empty object", the unknown object $\mathrm{x}$ can be interpreted as the distribution of the radiation passing through the SLM. The entries of $\Phi$ represent the transmission coefficients of the cells of the SLM, i.e. one cell for each projection of a micromirror. The linear and real valued model $\mathrm{x}=\Phi^{-1} \cdot \mathrm{y}$, employed so far as reconstruction algorithm, assumes that the sum of the radiation passing through each cell of the SLM weighted by the transmission coefficients yields the signal $(\mathrm{y})$, which is received by the detector. This model, however, does not sufficiently reflect the behaviour of the system.

Therefore, the model is extended to consider wave propagation of a certain wavelength radiation as well as the geometry of the setup. The simulated signal values of this model are calculated by a Kirchhoff integral (see [3]), which is discretised in order to solve it numerically. The Kirchhoff integral calculates the amplitude of radiation originating from a point source, passing through an extended aperture of arbitrary shape and reassembling at a distinct point on a socalled screen. The SLM is modelled as the aperture of arbitrary shape, the detector as a point on the screen. The installed detector $(\mathrm{Rx})$ is equipped with a horn antenna that responds non-uniformly to radiation from different angles. This property is modelled by weighting rays arriving at the screen depending on their incident angle. Overall, the setup is modelled by more than 15 parameters. 


\section{MEASurement And SimUlation Results}

The SPC setup is analysed by observing the detector signals for several sets of spatial filter configurations. Each filter set contains simple geometric features, such as straight lines, wedges, squares or rings of varying size. In Fig. 3 the measured and predicted detector signals for two filter sets of varying structure sizes are shown.
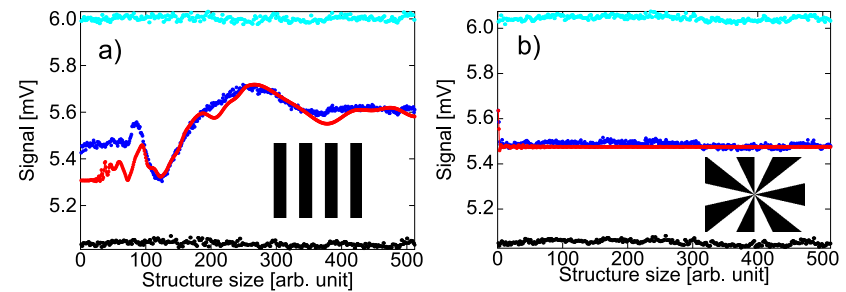

Fig. 3. The dark blue symbols represent the measured signal, while the red symbols are predicted values. Cyan and black symbols indicate the measured reference level of a fully open or closed spatial filter. The inset shows a representative for the filter set, i.e. the SLM mask. a) The filter set represents vertical grids, structure size equals line width in number of micromirrors. b) The filter set represents Siemens stars, structure size equals number of segment pairs (black, white).

The parameter-values of the model can be approximately derived from the setup itself. Those employed for the comparison with the measured signals have been found by systematically varying the values of the parameters and evaluating the correlation coefficients of the simulated and measured signals. A set of values was found that simultaneously provided correlation coefficients larger than $98 \%$ for all analysed sets of spatial filter configurations. Optimising the parameters for only a single set of spatial filter configurations can easily lead to overfitting and furthermore, it is likely to find meaningless models in the presence of correlated parameters. In such situations the parameters tend not to reflect the physical system. Therefore it is important to find a set of parameter-values that agrees with all analysed spatial filter sets.

\section{DisCuSSION AND OUTLOOK}

As Fig. 3 shows, specific filter sets do not alter the detected signal. Other filter sets, in contrast, result in distinct features in the measured and simulated signals. It is shown that with minor differences, the predicted values follow the dominant features of the measured signals for all filter sets analysed. This indicates, that the model correctly describes a major part of the actual system behaviour and can be used to predict the response of an SLM based setup in different applications. Following this interpretation, the circular artefact is an interference pattern and its shape depends on the geometry of the setup as well as the wavelength of the radiation source.

In the experimental setup the source in combination with the collimating lens yields a quasi-plane wave at the SLM. Unlike the experimental setup, the currently presented model uses a uniformly radiating point source in a far distance, which results in a similar quasi-plane wave at the aperture. To better reflect the physical setup, optical elements, like lenses or mirrors, should be introduced to the model. Furthermore, an antenna profile should be implemented for the source.

\section{ACKNOWLEDGEMENTS}

J. Hieronymus gratefully acknowledges the support by the Helmholtz Research School on Security Technologies.

This work has been supported by the project "Compressed Sensing for Terahertz Body Scanners" in the Priority Programme SPP1798 (CoSIP) of the Deutsche

Forschungsgemeinschaft.

\section{REFERENCES}

[1]. J. Hieronymus, S. Augustin, and H.-W. Hübers, "Characterization of a THz SLM and its application for improved high resolution THz imaging," 40th International Conference on Infrared, Millimeter, and Terahertz waves (IRMMW-THz), 2015, pp. 1-2, 2015.

[2]. S. Augustin, J. Hieronymus, P. Jung, and H.-W. Hübers. "Compressed sensing in a fully non-mechanical $350 \mathrm{GHz}$ imaging setting," Journal of Infrared, Millimeter, and Terahertz Waves, 36(5), pp. 496-512, 2015.

[3]. M. Born and E. Wolf, "Principles of Optics: Electromagnetic Theory of Propagation, Interference and Diffraction of Light," Pergamon Press, 1970 